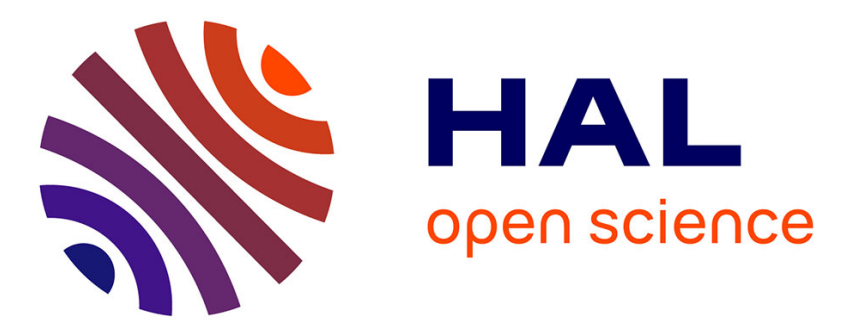

\title{
Development of Analytical Eco-Driving Cycles for Electric Vehicles
}

\author{
Luis Alfredo Wulf Ribelles, Kristan Gillet, Guillaume Colin, Yann
}

Chamaillard, Antoine Simon, Cédric Nouillant

\section{- To cite this version:}

Luis Alfredo Wulf Ribelles, Kristan Gillet, Guillaume Colin, Yann Chamaillard, Antoine Simon, et al.. Development of Analytical Eco-Driving Cycles for Electric Vehicles. European Control Conference ECC 2021, Jun 2021, Rotterdam (virtual), Netherlands. hal-03279349

\section{HAL Id: hal-03279349 \\ https://hal.science/hal-03279349}

Submitted on 6 Jul 2021

HAL is a multi-disciplinary open access archive for the deposit and dissemination of scientific research documents, whether they are published or not. The documents may come from teaching and research institutions in France or abroad, or from public or private research centers.
L'archive ouverte pluridisciplinaire HAL, est destinée au dépôt et à la diffusion de documents scientifiques de niveau recherche, publiés ou non, émanant des établissements d'enseignement et de recherche français ou étrangers, des laboratoires publics ou privés. 


\title{
Development of Analytical Eco-Driving Cycles for Electric Vehicles
}

\author{
L. A. Wulf Ribelles ${ }^{a}$, K. Gillet ${ }^{a}$, G. Colin ${ }^{a}$, Y. Chamaillard ${ }^{a}$, A. Simon ${ }^{b}$ and C. Nouillant ${ }^{b}$
}

\begin{abstract}
This paper presents the development of analytical solutions for the computation of Eco-Driving cycles for electric vehicles. The task of defining an Eco-Driving strategy is formulated as an Optimal Control Problem aiming to minimize the energy consumed during a trip subject to input and speed constraints. Here, the final time of the driving mission is set as a free parameter and only the relevant terms for the optimization are taken into account. The problem is solved using Pontryagin's Minimum Principle in a systematic way, allowing the derivation of closed-form expressions for the different (un)constrained solutions. The results obtained with the proposed approach are compared to the optimal solution given by Dynamic Programming, where a minor deviation from the optimal consumption is achieved while drastically reducing the computation time of the solution.
\end{abstract}

\section{INTRODUCTION}

With the need to reduce greenhouse emissions, the improvement of vehicle energy efficiency has become a relevant field of research for the automotive industry during the last decades due to its role as a major energy consumer worldwide. In this context, the combination of energy management strategies (EMS), legal regulations and driver assistance technology led to the concept of Eco-Driving (ED) [1], which has become one of the most promising ways to improve the energetic performance of road vehicles. Put briefly, it describes an energy efficient driving behaviour based on the trip information and previous studies to assess its potential have reported up to $20 \%$ of energy savings [2], [3]. Together with energy efficient strategies, governments and industry are promoting the expansion of vehicle electrification to achieve a lower number of petrol-based cars on the roads and mitigate vehicle $\mathrm{CO}_{2}$ emissions. A market share of $30 \%$ is expected for electric vehicles in the following years [4] and motivates the development of EMS for electric vehicles (EV).

The definition of an ED strategy can be formulated as an Optimal Control Problem (OCP) [5] and different methods can be considered to solve it, namely, Dynamic Programming (DP), Static Optimization (SO) and Pontryagin's Minimum Principle (PMP). Among the three, DP has been the most common technique due to its ability to handle any kind of OCP while guaranteeing global optimality of the solution. In [6] and [7], DP was used to obtain the optimal speed profiles for various driving missions and evaluate the tradeoff between model complexity and quality of the solution. The main restriction of DP is caused by the so-called "Curse of Dimensionality", which limits the number of

${ }^{a}$ L. A. Wulf Ribelles, K. Gillet, G. Colin and Y. Chamaillard are with Laboratoire PRISME, Université d'Orléans, 45072 Orléans, France. E-mail: luis-alfredo.wulf-ribelles@univ-orleans.fr

${ }^{b}$ A. Simon and C. Nouillant are with Groupe PSA, Division for Research \& Innovations, 78955 Carrières-sous-Poissy, France. states and decision variables that can be considered in the problem despite possible distance-based or receding horizon reformulations to reduce the problem complexity [8].

Nowadays, methods based on SO are more prominent due to their popularity in Model Predictive Control frameworks and the improved computational power in today's devices. Nonetheless, the ED problem is generally non-convex and requires special treatments in order to be solved efficiently, e.g., convexification methods, in combination with fast stateof-art solvers [9]-[11]. For instance, a reformulation dealing with the loss of convexity during discretization to obtain global optimal speed trajectories was developed in [9] while proposing a sequential quadratic programming algorithm to solve the problem but, despite an easier incorporation of constraints and road grade information, this approach can still be time consuming for long driving cycles or when different position dependent constraints are imposed.

Alternatively, PMP has been used to tackle computation complexity by deriving analytical solutions that provide a better insight into how energy savings can be obtained while allowing for an easier implementation in real time. Although the non-linear characteristics of the ED problem often require the use of numerical methods to obtain a solution, it is possible to arrive at a Boundary Value Problem (BVP) that can be solved in closed-form if sufficiently simple models are considered (a general overview of PMP-based solutions can be found in [5] and [6]). Besides these examples, the combination of speed and input constraints in ED for EVs has not been explored in the literature and, to the Authors' knowledge, only a few examples of state-constrained cases are available [6], [12], [13]. Therefore, the contributions of this paper attain the development of free final time analytical solutions to obtain energy efficient driving profiles in the presence of input and speed constraints. These solutions provide a wider range of driving patterns in comparison to the aforementioned studies while handling situations where the trip duration is not known. Moreover, a tuning strategy for the computation of the Eco-Driving cycles is adopted to provide an alternative to the DP methods in [7], [8].

The paper is organized as follows: Section II presents the vehicle model considered in this work. An OCP formulation for ED is provided in Section III. Section IV includes the analytical solutions to the OCP for different combinations of active constraints. Two simple case studies to illustrate the behaviour of the closed-form solutions are presented in Section V. After this, the considerations for the computation of ED cycles are described in Section VI followed by the simulation results in Section VII. Finally, Section VIII presents some conclusions and perspectives for future work. 


\section{VEHICLE MODEL}

In this section, we introduce the modeling approach used in this paper and present the different simplifications and assumptions considered for the analytical computations. (The vehicle data and model parameters are confidential).

\section{A. Vehicle Dynamics}

The motion of the vehicle is modeled in a longitudinal direction following Newton's $2^{\text {nd }}$ law:

$$
\begin{aligned}
& \dot{s}(t)=v(t) \\
& \dot{v}(t)=\frac{1}{m}\left(F_{t}(t)-F_{r}(t)\right)
\end{aligned}
$$

where $F_{t}$ is the traction force to propel the vehicle, $F_{r}$ is the sum of resistance forces and $m$ is the total mass of the vehicle including the inertia of the rotating parts in the driveline.

The resistance forces in (1b) are represented by a quadratic polynomial of the vehicle speed [7], [8]

$$
F_{r}(t)=a_{0}+a_{1} v(t)+a_{2} v(t)^{2}
$$

where $a_{1}$ and $a_{0}=\bar{a}+m g \sin (\theta(s))$ describe the rolling and road slope resistance, respectively, and $a_{2}$ captures the aerodynamic resistance. These coefficients are identified for the particular vehicle in this work considering a flat road.

\section{B. Transmission}

In this model, the relation between the traction force $F_{t}$ and the torque of the electric motor assuming no slip at wheels is described as

$$
F_{t}=\left(\eta_{t} R_{t} T(t)\right) / r_{\text {tire }}
$$

with $\eta_{t}$ being a constant transmission efficiency, $R_{t}$ the transmission ratio, $T$ the motor torque and $r_{\text {tire }}$ the wheel radius. Here, a positive torque propels the vehicle and negative values correspond to regenerative braking. Similarly, the relation between the angular speed of the motor and the vehicle speed is given by $v(t)=\left(\omega(t) r_{\text {tire }}\right) / R_{t}$.

\section{Vehicle Dynamics Simplifications}

As expressed in [6], the non-linear characteristics of the vehicle dynamics have a large impact on complexity when used to generate closed-form solutions. The velocity dependent terms in (1b) were therefore neglected in this study, given that this approximation tends to be valid for urban driving scenarios [6], [12], [13]. Moreover, the vehicle dynamics are written in terms of acceleration and a flat road is assumed, resulting in $\dot{v}(t)=u(t)-c_{0}$, with the acceleration input $u(t)=\left(\eta_{t} R_{t} T(t)\right) /\left(m r_{\text {tire }}\right)$ and $c_{0}=a_{0} / m$.

\section{Electric Motor}

In order to describe the energy consumption of the EV, the power losses of the battery are neglected to reduce the complexity of the model, giving $P_{b}(t)=P_{m}(t)+P_{a u x}$. Here, the power consumed by the electric motor is represented by

$$
P_{m}(u, v)=b_{1} v(t)+b_{2} u(t) v(t)+b_{3} u(t)^{2}
$$

where $b_{1}$ captures the friction losses, $b_{2}$ corresponds to a mechanical power equivalence and $b_{3}$ accounts for the
Ohmic losses, with $b_{\{1,2,3\}} \in \mathbb{R}_{>0}$. This representation is comparable to other models in the literature [6], [9] while maintaining its simplicity to perform analytic calculations. Here, the parameters of $P_{m}$ are identified from a quasi-static map of the electric machine that includes the power losses of the motor and the electronic devices.

In this regard, Fig. 1 shows the model accuracy with respect to the electric motor map, where the RMSE of the power predictions with model (4) is $0.85 \mathrm{~kW}$.

\section{ECO-DRIVING PROBLEM}

Now, the problem statement defined from the ED concept is presented along with the method employed to obtain the analytical solutions. In general, an Eco-Driving strategy aims to define the optimal speed profile that a vehicle should follow to minimize its energy consumption for a trip along a specific route $s(t) \in\left[s_{0}, s_{f}\right]$ in a given time $t \in\left[t_{0}, t_{f}\right]$ while respecting legal or situational driving restrictions, i.e., speed limits and traffic conditions [5]-[13].

\section{A. Optimal Control Problem Formulation}

The task of defining an ED strategy can be formulated as the following continuous-time optimal control problem

$$
\begin{array}{ll}
\min _{\left\{u(t), s(t), v(t), t_{f}\right\}} & \int_{t_{0}}^{t_{f}}(L(u, v)+\beta) d t \\
\text { subject to } & \\
& \dot{s}(t)=v(t) \\
& \dot{v}(t)=u(t)-c_{0} \\
& s\left(t_{0}\right)=s_{0}, \quad s\left(t_{f}\right)=s_{f} \\
& v\left(t_{0}\right)=v_{0}, \quad v\left(t_{f}\right)=v_{f} \\
& v(t) \leq v_{\max } \\
& -u_{\min } \leq u(t) \leq u_{\max }
\end{array}
$$

where the term $\beta$ prioritizes the duration of the trip over the energy savings, given that only certain applications have predefined arrival times, e.g., schedules of city buses [10].

Based on the study [7], only the power consumed by the electric motor is considered in (5a) since the auxiliaries had a minor influence in the optimization of the vehicle speed, thus $L(u, v)=P_{m}(u, v)$; although $P_{a u x}$ could be linked to $\beta$ in low charge scenarios. Additionally, $v_{\max }, u_{\max }$ and $u_{\min }$ are considered as constant speed and input limits, respectively.

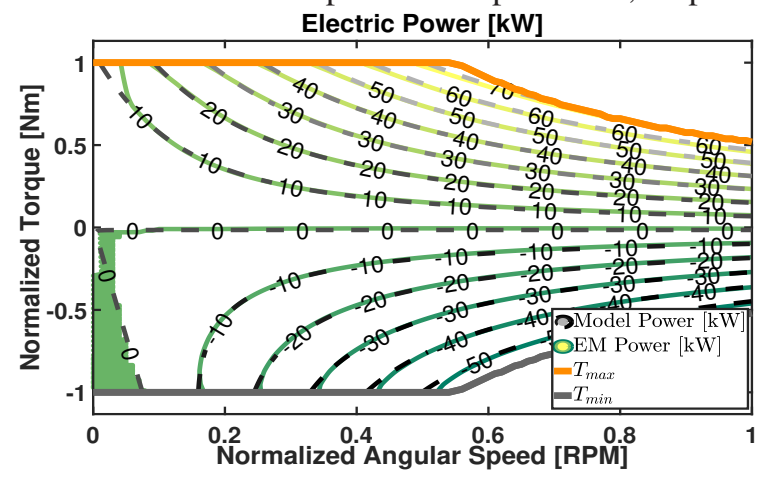

Fig. 1: Normalized electric motor power map vs. model power predictions as a function of torque and angular speed. 
Even though the solution to (5) can be obtained through different methods, e.g., DP, SO or PMP, we focus on a Pontryagin's Minimum Principle approach to derive a set of analytical solutions that provides a straightforward implementation when including position-based information while having a simple interpretation of the speed behaviour. Nonetheless, it should be noted that fewer terms involved in the OCP can lead to simpler closed-form expressions.

To this end, the previous OCP can be reformulated to obtain more compact expressions by removing the terms that can be solved in advance, considering that conditions (5d) and (5e) are fixed as explained in [6], [9]. A compact representation can be obtained by substituting the simplified speed dynamics (5c) in the consumption model and then integrating the resulting cost function $\mathcal{J}=$ $\int_{t_{0}}^{t_{f}}\left(b_{1} v(t)+b_{2}\left(\dot{v}(t)+c_{0}\right) v(t)+b_{3}\left(\dot{v}(t)+c_{0}\right)^{2}+\beta\right) d t$ over the boundary conditions $(5 \mathrm{~d})$ and $(5 \mathrm{e})$, leaving only the terms contributing to the energy saving along the trip

$$
\begin{aligned}
\mathcal{J} & =\left(b_{1}+b_{2} c_{0}\right)\left(s_{f}-s_{0}\right)+\frac{1}{2} b_{2}\left(v_{f}^{2}-v_{0}^{2}\right) \\
& +2 b_{3} c_{0}\left(v_{f}-v_{0}\right)+\int_{t_{0}}^{t_{f}}\left(b_{3} \dot{v}(t)^{2}+b_{3} c_{0}^{2}+\beta\right) d t
\end{aligned}
$$

Despite being constant, the last two terms in the integral are kept since $t_{f}$ is an unknown parameter. With this new cost and substituting back the speed dynamics (5c), the continuous-time Eco-Driving OCP to be solved is given by

$$
\min _{\left\{u(t), s(t), v(t), t_{f}\right\}} \int_{t_{0}}^{t_{f}}(\underbrace{b_{3}\left(u(t)-c_{0}\right)^{2}+b_{3} c_{0}^{2}}_{\tilde{L}(u)}+\beta) d t
$$

subject to

$$
\text { Constraints }(5 b)-(5 g)
$$

\section{Analytical Solution to the ECO-Driving OCP}

In this section, the analytical solutions to problem (7) and the methodology used for their derivation are presented.

\section{A. Pontryagin's Minimum Principle and Solution Approach}

We begin by defining the Hamiltonian

$$
\mathcal{H}=\tilde{L}(u)+\beta+\lambda_{s} v+\lambda_{v}\left(u-c_{0}\right)
$$

Following [14], the equations describing the state and costate dynamics are obtained by taking the partial derivatives of (8) with respect to each variable such that

$$
\begin{aligned}
\frac{d \mathcal{H}}{d \lambda_{s}} & =\dot{s}=v \\
\frac{d \mathcal{H}}{d \lambda_{v}} & =\dot{v}=u-c_{0} \\
-\frac{d \mathcal{H}}{d s} & =\dot{\lambda}_{s}=0 \\
-\frac{d \mathcal{H}}{d v} & =\dot{\lambda}_{v}=-\lambda_{s}
\end{aligned}
$$

In the absence of any input or speed constraint, the optimal input that minimizes the Hamiltonian is given by

$$
\frac{d \mathcal{H}}{d u}=2 b_{3}\left(u-c_{0}\right)+\lambda_{v}=0 \rightarrow u^{*}=-\frac{\lambda_{v}}{2 b_{3}}+c_{0}
$$

which is substituted in (9) to obtain a Two Point Boundary Value Problem (TPBVP). Furthermore, one can guarantee that (10) is optimal if $d^{2} \mathcal{H} / d u^{2}=2 b_{3} \geq 0$, which is already satisfied by the definition of the consumption model.

The following step is the integration of the resulting system of differential equations but, in order to obtain the solution to (7) in a compact and systematic way, the system (9) with (10) is written in matrix form with the help of an augmented state vector, $x=\left\{s, v, 1, \lambda_{s}, \lambda_{v}, 0\right\}^{\top}$, giving the system $\dot{x}(t)=H x(t)$. Furthermore, the presence of input and state constraints transforms the previous autonomous system into a time-varying linear system $\dot{x}(t)=H(t) x(t)$ with piece-wise constant dynamics described by

$$
H(t)= \begin{cases}H_{a}, & \text { if } t_{0} \leq t<t_{a} \\ H_{b}, & \text { if } t_{a} \leq t<t_{b} \\ \vdots & \\ H_{F}, & \text { if } t_{k} \leq t \leq t_{f}\end{cases}
$$

where $t_{0}<t_{a}<t_{b} \ldots t_{k}<t_{f}$ are switching times determined by the active constraints defining different driving modes.

First, consider the presence of input constraints given that (10) is limited by the maximum (minimum) torque capacity of the motor. Thus, the constrained optimal input is given by

$$
u(t)^{*}= \begin{cases}u_{\max }, & \text { if } u(t)^{*} \geq u_{\max } \\ -\frac{\lambda_{v}}{2 b_{3}}+c_{0}, & \text { if }-u_{\min }<u(t)^{*}<u_{\max } \\ -u_{\min }, & \text { if } u(t)^{*} \leq-u_{\min }\end{cases}
$$

In the case of pure state inequality constraints [14], the Hamiltonian is modified by adjoining the constraints in an indirect approach to form a Lagrangian $\mathcal{L}=\mathcal{H}+\mu g^{(1)}$ satisfying the tangency conditions

$$
g(v)=0 \text { and } g^{(1)}(v)=\frac{d}{d t}(g(v)) \leq 0
$$

where $g(v):=v(t)-v_{\max }$ and $\mu$ is a lagrange multiplier satisfying the complementary slackness condition $\mu g=0$ with $\mu \geq 0$ for all $t \in\left[t_{0}, t_{f}\right]$. Since this OCP only considers first order indirect constraints, the speed limit is active during a single interval, e.g., $\left[t_{a}, t_{b}\right]$, and the continuity of the state trajectory at the entry point imposes the jump conditions

$$
\begin{aligned}
\lambda_{s}\left(t_{a}^{-}\right) & =\lambda_{s}\left(t_{a}^{+}\right) \\
\lambda_{v}\left(t_{a}^{-}\right) & =\lambda_{v}\left(t_{a}^{+}\right)+\pi_{v} \frac{d}{d v}\left(g\left(v^{*}, t_{a}^{+}\right)\right) \\
\mathcal{H}\left(t_{a}^{-}\right) & =\mathcal{H}\left(t_{a}^{+}\right)
\end{aligned}
$$

where the multiplier $\pi_{v}$ satisfies $\pi_{v} g=0$ and $\pi_{v} \geq 0$.

By incorporating these conditions into the switching dynamics (11), the solution to the LTV Hamiltonian system can be easily obtained via the matrix exponential, such that

$$
x\left(t_{f}\right)=e^{H_{F}\left(t_{f}-t_{k}\right)} \ldots e^{H_{b}\left(t_{b}-t_{a}\right)} e^{H_{a}\left(t_{a}-t_{0}\right)} x\left(t_{0}\right)
$$

where $x\left(t_{0}\right)=\left\{s_{0}, v_{0}, 1, \lambda_{s, 0}, \lambda_{v, 0}, 0\right\}^{\top}$ and the particular driving mode sequence is defined by the active constraints, going from an unconstrained to a fully constrained solution.

Finally, the trip duration is determined by the transversal condition $\mathcal{H}_{t_{f}}:=\left.\mathcal{H}\right|_{t=t_{f}}=0$, given that the Hamiltonian must be constant since it does not depend explicitly on time [14] and where the trip time varies according to the value of $\beta$. Furthermore, we consider $t_{0}=s_{0}=0$ in order to simplify the derivations and the resulting expressions. 


\section{B. Unconstrained Solution}

In the absence of any input or speed constraint, the analytical solution is given by a single mode denominated Partial Acceleration (PA) resulting from $x\left(t_{f}\right)=e^{H_{p}\left(t_{f}\right)} x(0)$ and where the only unknowns are the co-state initial conditions (Co-ICs) and the trip duration $t_{f}$. The speed trajectory in this driving mode coincides with the parabolic profile reported in the literature [6], [12], [13] and is given by the quadratic equation of time:

$$
v(t)=\left(\frac{\lambda_{s, 0}}{4 b_{3}}\right) t^{2}+\left(-\frac{\lambda_{v, 0}}{2 b_{3}}\right) t+v_{0}
$$

where

$$
\begin{aligned}
\lambda_{s, 0} & =\frac{12 b_{3}\left(t_{f} v_{f}-2 s_{f}+t_{f} v_{0}\right)}{t_{f}{ }^{3}} \\
\lambda_{v, 0} & =\frac{4 b_{3}\left(t_{f} v_{f}-3 s_{f}+2 t_{f} v_{0}\right)}{t_{f}^{2}} \\
\mathcal{H}_{t_{f}} & =\frac{\left(\beta+b_{3} c_{0}^{2}\right) t_{f}^{4}-b_{3}\left(6 s_{f}-2\left(v_{f}+v_{0}\right) t_{f}\right)^{2}+4 b_{3} v_{f} v_{0} t_{f}^{2}}{t_{f}^{4}}
\end{aligned}
$$

Here, the Co-ICs are given by the conditions $v\left(t_{f}\right)=v_{f}$, $s\left(t_{f}\right)=s_{f}$ and the real positive solutions of $\mathcal{H}_{t_{f}}=0$ define possible optimal final times. The selection of $t_{f}$ depends on the boundary conditions of each particular trip.

\section{Speed Constrained Solution}

This scenario considers a situation where the speed limit is reached but $u^{*}$ is not affected by any of the input constraints and incorporates a second driving mode in the solution denominated Constant Speed (CS). This results in two additional conditions appearing at the entry time of the constant speed phase, i.e., $v\left(t_{a}\right)=v_{\max }$ and $\dot{v}\left(t_{a}\right)=0$, that need to be satisfied with (13) and (14).

Now, the speed constrained solution is composed by two switching times and a driving mode sequence $P A-C S-P A$, i.e., $x\left(t_{f}\right)=e^{H_{p}\left(t_{f}-t_{b}\right)} e^{H_{c}\left(t_{b}-t_{a}\right)} e^{H_{p}\left(t_{a}\right)} x(0)$, where the Co-ICs and the entry, exit and final times are unknown. This results in a speed profile of the form:

$$
v(t)= \begin{cases}\left(\frac{\lambda_{s, 0}}{4 b_{3}}\right) t^{2}+\left(-\frac{\lambda_{v, 0}}{2 b_{3}}\right) t+v_{0}, & \text { if } t \in\left[0, t_{a}\right) \\ v_{\max }, & \text { if } t \in\left[t_{a}, t_{b}\right) \\ \left(\frac{\lambda_{s, 0}}{4 b_{3}}\right) t^{2}+\left(\frac{\lambda_{s, 0}\left(t_{a}-t_{b}\right)-\lambda_{v, 0}}{2 b_{3}}\right) t+\tilde{v}_{b}, & \text { if } t \in\left[t_{b}, t_{f}\right]\end{cases}
$$

where $\tilde{v}_{b}$, the Co-ICs and switching times are defined as

$$
\begin{aligned}
& \tilde{v}_{b}=v_{\max }+\frac{\lambda_{v, 0} t_{b}}{2 b_{3}}+\frac{\lambda_{s, 0} t_{b}\left(t_{b}-2 t_{a}\right)}{4 b_{3}} \\
& \lambda_{s, 0}=-\frac{4 b_{3}\left(v_{\max }-v_{0}\right)}{t_{a}^{2}} \\
& \lambda_{v, 0}=-\frac{4 b_{3}\left(v_{\max }-v_{0}\right)}{t_{a}} \\
& t_{a}=\frac{3 t_{f} v_{\max }-3 s_{f}}{v_{\max }-v_{0}-v_{f} \sigma+v_{\max } \sigma} \\
& t_{b}=t_{f}+\frac{\left(3 t_{f} v_{\max }-3 s_{f}\right)\left(v_{f}-v_{\max }\right)}{\left(v_{f}-v_{\max }\right)^{2}+\sigma\left(v_{\max }-v_{0}\right)^{2}} \\
& t_{f}=\frac{2 \beta s_{f}+2 b_{3} c_{0}^{2} s_{f}+\sqrt{b_{3}} v_{\max } \sqrt{b_{3} c_{0}^{2}+\beta} \sqrt{-\frac{16 \gamma}{9 v_{\max }}}}{2\left(b_{3} v_{\max } c_{0}{ }^{2}+\beta v_{\max }\right)} \\
& \gamma=\left(v_{f}-v_{\max }\right)^{3}+\left(v_{0}-v_{\max }\right)^{3}+2 \sigma\left(v_{f}-v_{\max }\right)\left(v_{\max }-v_{0}\right)^{2}
\end{aligned}
$$

with $\sigma=\sqrt{-\left(v_{f}-v_{\max }\right) /\left(v_{\max }-v_{0}\right)}$. In this case, an explicit expression for the final time can be obtained since $\mathcal{H}_{t_{f}}=0$ is a second order equation of $t_{f}$ with only one feasible solution.

\section{Input Constrained Solution}

Other possible variant of the analytic solution considers the cases where one or both input limits are not respected by the $P A$ solution, requiring a new driving mode for each input constraint referred to as Maximum Acceleration (MA) and Maximum Deceleration (MD), respectively. Due to space limitations, only the solution with both input constraints active is presented, although closed-form solutions with a single active input limit have been obtained and included during the simulations presented in the following sections.

In this case, the input constraints do not require any special treatment before being incorporated in the Lagrangian and they can be included in a sequential manner [6] using the conditions $u\left(t_{a}\right)=u_{\max }$ and $u\left(t_{b}\right)=-u_{\min }$, such that $x\left(t_{f}\right)=e^{H_{d}\left(t_{f}-t_{b}\right)} e^{H_{p}\left(t_{b}-t_{a}\right)} e^{H_{a}\left(t_{a}\right)} x(0)$. This results in a sequence $M A-P A-M D$ with a speed trajectory following:

$$
v(t)= \begin{cases}\left(-c_{0}+u_{\max }\right) t+v_{0}, & \text { if } t \in\left[0, t_{a}\right) \\ \left(\frac{\lambda_{s, 0}}{4 b_{3}}\right) t^{2}+\left(-\frac{\lambda_{v, 0}}{2 b_{3}}\right) t+\tilde{v}_{a}, & \text { if } t \in\left[t_{a}, t_{b}\right) \\ \left(-c_{0}-u_{\min }\right) t+\tilde{v}_{b}, & \text { if } t \in\left[t_{b}, t_{f}\right]\end{cases}
$$

Here, the initial speed for each phase is given by

$$
\begin{aligned}
& \tilde{v}_{a}=v_{0}+t_{a}\left(-c_{0}+u_{\max }\right)+\frac{\lambda_{v, 0} t_{a}}{2 b_{3}}-\frac{\lambda_{s, 0} t_{a}{ }^{2}}{4 b_{3}} \\
& \tilde{v}_{b}=\tilde{v}_{a}+t_{b}\left(c_{0}+u_{\text {min }}\right)-\frac{\lambda_{v, 0} t_{b}}{2 b_{3}}+\frac{\lambda_{s, 0} t_{b}{ }^{2}}{4 b_{3}}
\end{aligned}
$$

and the expressions for the unknown variables are

$$
\begin{aligned}
& \lambda_{s, 0}=-\frac{\sqrt{3} b_{3}\left(u_{\max }+u_{\min }\right)^{2}}{3 \sqrt{\phi}} \\
& \lambda_{v, 0}=\frac{\lambda_{s, 0}\left(v_{f}-v_{0}+c_{0} t_{f}+t_{f} u_{\min }\right)+b_{3}\left(\left(c_{0}+u_{\min }\right)^{2}-\left(c_{0}-u_{\max }\right)^{2}\right)}{u_{\min }+u_{\max }} \\
& t_{a}=\frac{v_{f}-v_{0}+c_{0} t_{f}+t_{f} u_{\min }-\sqrt{3} \sqrt{\phi}}{u_{\min }+u_{\max }} \\
& t_{b}=\frac{v_{f}-v_{0}+c_{0} t_{f}+t_{f} u_{\min }+\sqrt{3} \sqrt{\phi}}{u_{\min }+u_{\max }} \\
& \phi=-\left(v_{f}-v_{0}\right)^{2}+t_{f}^{2}\left(u_{\max }-c_{0}\right)\left(u_{\min }+c_{0}\right)+2 c_{0} t_{f}\left(v_{0}-v_{f}\right)+ \\
& \left(2 t_{f} v_{f}-2 s_{f}\right) u_{\max }+\left(2 t_{f} v_{0}-2 s_{f}\right) u_{\min } \\
& t_{f}=-\frac{\sqrt{3} \sqrt{\frac{p}{q}} \sqrt{u_{\min }+u_{\max }}\left(\beta+b_{3}\left(c_{0}\left(u_{\max }-u_{\min }\right)+u_{\min } u_{\max }\right)\right)}{\left(c_{0}+u_{\min }\right)\left(c_{0}-u_{\max }\right)} \\
& +\frac{c_{0} v_{0}-c_{0} v_{f}+u_{\max } v_{f}+u_{\min } v_{0}}{\left(c_{0}+u_{\min }\right)\left(c_{0}-u_{\max }\right)}
\end{aligned}
$$

The terms $p$ and $q$ in (18i) are omitted for space reasons. Note that separate final time conditions $H_{t_{f}}=0$ are obtained when only one of the input constraints is active.

\section{E. Fully Constrained Solution}

To conclude, an input and speed constrained solution is obtained by combining all the previous cases to define a driving mode sequence $M A-P A-C S-P A-M D$. Even though multiple variations of this solution can exist depending on the constraints that become active during a particular trip, only the case where all the driving modes appear in the trajectory is presented in this paper, i.e., a switching sequence $x\left(t_{f}\right)=$ $e^{H_{d}\left(t_{f}-t_{d}\right)} e^{H_{p}\left(t_{d}-t_{c}\right)} e^{H_{c}\left(t_{c}-t_{b}\right)} e^{H_{p}\left(t_{b}-t_{a}\right)} e^{H_{a}\left(t_{a}\right)} x(0)$. This gives the analytical speed profile: 

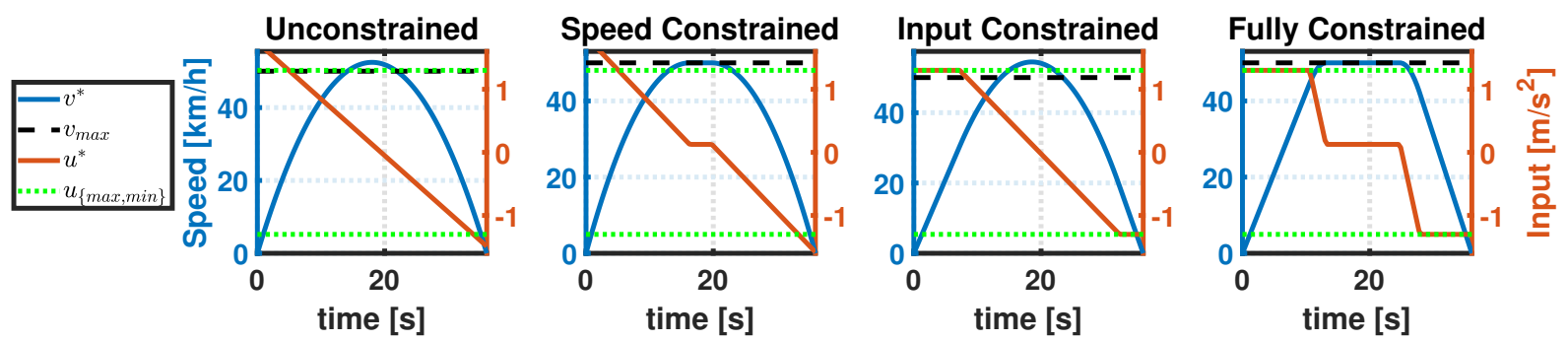

Fig. 2: Optimal speed and control input for the analytical solutions in Section IV-B to IV-E in a single trip of $s_{f}=350[m]$ with $v_{0}=v_{f}=0[\mathrm{~km} / \mathrm{h}], v_{\max }=50[\mathrm{~km} / \mathrm{h}], u_{\{\max , \min \}}=1.3\left[\mathrm{~m} / \mathrm{s}^{2}\right]$ and $t_{f}=36.02[\mathrm{~s}]\left(\beta=2 \times 10^{4}\right)$.

$$
v(t)= \begin{cases}\left(-c_{0}+u_{\max }\right) t+v_{0}, & \text { if } t \in\left[0, t_{a}\right) \\ \left(\frac{\lambda_{s, 0}}{4 b_{3}}\right) t^{2}+\left(-\frac{\lambda_{v, 0}}{2 b_{3}}\right) t+\tilde{v}_{a}, & \text { if } t \in\left[t_{a}, t_{b}\right) \\ v_{\max }, & \text { if } t \in\left[t_{b}, t_{c}\right) \\ \left(\frac{\lambda_{s, 0}}{4 b_{3}}\right) t^{2}+\left(\frac{\lambda_{s, 0}\left(t_{a}-t_{b}\right)-\lambda_{v, 0}}{2 b_{3}}\right) t+\tilde{v}_{c}, & \text { if } t \in\left[t_{c}, t_{d}\right) \\ \left(-c_{0}-u_{\min }\right) t+\tilde{v}_{d}, & \text { if } t \in\left[t_{d}, t_{f}\right]\end{cases}
$$

again, the initial speeds can be explicitly defined as

$$
\begin{aligned}
& \tilde{v}_{a}=v_{0}+t_{a}\left(-c_{0}+u_{\max }\right)+\frac{\lambda_{v, 0} t_{a}}{2 b_{3}}-\frac{\lambda_{s, 0} t_{a}{ }^{2}}{4 b_{3}} \\
& \tilde{v}_{c}=\tilde{v}_{\text {max }}+\frac{\lambda_{v, 0} t_{c}}{2 b_{3}}+\frac{\lambda_{s, 0} t_{c}\left(t_{c}-2 t_{b}\right)}{4 b_{3}} \\
& \tilde{v}_{d}=\tilde{v}_{c}+t_{d}\left(c_{0}+u_{\min }\right)+\frac{\lambda_{v, 0} t_{d}}{2 b_{3}}+\frac{\lambda_{s, 0} t_{d}\left(t_{d}-2\left(t_{c}+t_{b}\right)\right)}{4 b_{3}}
\end{aligned}
$$

and the switching times and Co-ICs are

$$
\begin{aligned}
& \lambda_{s, 0}=-\frac{b_{3} c_{0}{ }^{2}+\beta}{v_{\max }} \\
& \lambda_{v, 0}=-\frac{\lambda_{s, 0}\left(v_{\max }-v_{0}\right)-b_{3}\left(c_{0}-u_{\max }\right)^{2}}{\left(c_{0}-u_{\max }\right)} \\
& t_{a}=-\frac{\lambda_{s, 0}\left(v_{\max }-v_{0}\right)+b_{3}\left(c_{0}-u_{\max }\right)^{2}}{\lambda_{s, 0}\left(c_{0}-u_{\max }\right)} \\
& t_{b}=-\frac{\lambda_{s, 0}\left(v_{\max }-v_{0}\right)-b_{3}\left(c_{0}-u_{\max }\right)^{2}}{\lambda_{s, 0}\left(c_{0}-u_{\max }\right)} \\
& t_{c}=\frac{\lambda_{s, 0}\left(v_{f}-v_{\max }+t_{f}\left(c_{0}+u_{\min }\right)\right)+b_{3}\left(c_{0}+u_{\min }\right)^{2}}{\lambda_{s, 0}\left(c_{0}+u_{\min }\right)} \\
& t_{d}=\frac{\lambda_{s, 0}\left(v_{f}-v_{\max }+t_{f}\left(c_{0}+u_{\min }\right)\right)-b_{3}\left(c_{0}+u_{\min }\right)^{2}}{\lambda_{s, 0}\left(c_{0}+u_{\min }\right)} \\
& t_{f}=-\frac{\psi}{6 v_{\max }\left(c_{0}+u_{\min }\right)\left(b_{3} c_{0}^{2}+\beta\right)^{2}\left(c_{0}-u_{\max }\right)}
\end{aligned}
$$

where the term $\psi$ is a function of the problem parameters but the expression is omitted due to its length. The main difference in this solution is related to the derivations of the final time and the position co-state, since the resulting transversal condition $\mathcal{H}_{t_{f}}=0$ does not depend on the final time and condition $s\left(t_{f}\right)=s_{f}$ is linear in $t_{f}$.

\section{F. Sub-Problem Solutions}

As mentioned before, several variations in the driving mode sequences defining the optimal speed profiles can appear depending on the trip at hand. Therefore, closed-form solutions have been derived for the trips that do not present all the driving modes in the cases previously mentioned. This includes solutions where either the initial or final speed conditions are equal to the speed limit, i.e., $v(0)=v_{\max }$ or $v\left(t_{f}\right)=v_{\max }$, since these situations are not compatible with the expressions in Section IV-C and IV-E. To summarize, the

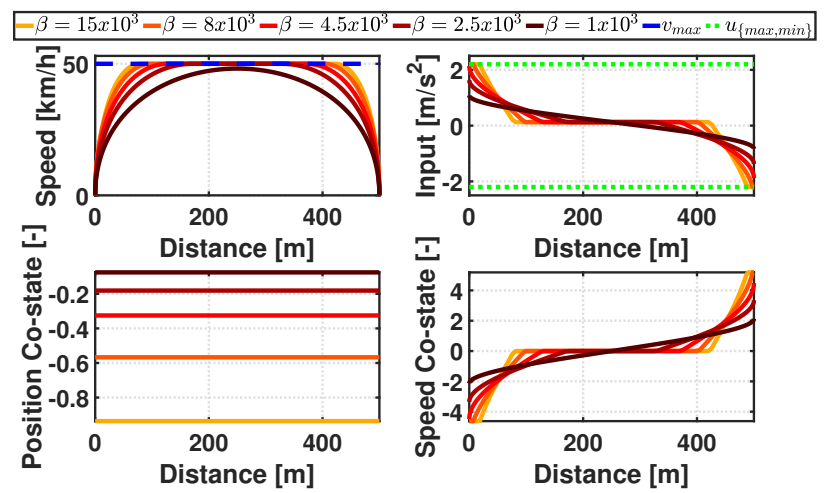

Fig. 3: Optimal speed, control input, and (scaled) co-state trajectories for a single trip of $s_{f}=500 \mathrm{~m}$ with $v_{0}=v_{f}=0$ and a decreasing sequence of penalization values $\beta$.

driving mode sequences defined in this work can be grouped in 3 categories:

- Accelerations: (MA-)PA-CS and (MA-)PA

- Decelerations: $C S-P A(-M D)$ and $P A(-M D)$

- Eco-Trips: (MA-)PA(-MD) and (MA-)PA-CS-PA(-MD)

where the active constraints are successively verified to select the appropriate driving mode sequence and each solution has its own Co-ICs, switching and final time expressions.

\section{Simple Case Studies}

This section presents the simulations during two simple case studies to show the capabilities of the different analytical solutions. The case studies consider single Eco-Trips between two stops covering a distance of 350 and $500[\mathrm{~m}]$, respectively, and with a speed limit of $50[\mathrm{~km} / \mathrm{h}]$. First, an illustrative example of the trajectories generated with the solutions in Section IV-B to IV-E is presented in Fig. 2. Here, $t_{f}$ is fixed based on the fully constrained solution and the input constraints are tightened for visualization purposes, since the third speed profile tries to follow an unconstrained behaviour while compensating for the limited acceleration and deceleration patterns. In the second case, a longer trip with free final time is considered, where the penalty term $\beta$ is modified to force the presence of the different driving modes in the speed trajectories. As seen in Fig. 3, the reduction of the final time has a strong influence on the final driving mode sequence, going from an unconstrained parabolic profile with $t_{f}=56.03[s]$ to a fully constrained profile that takes $42.70[s]$ to complete the trip. 

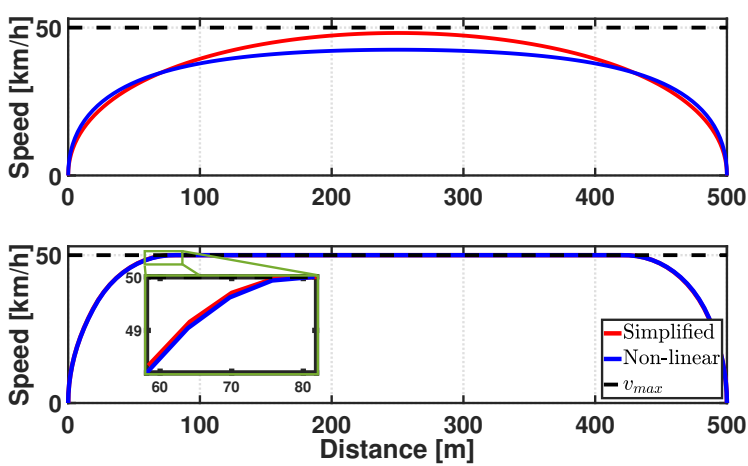

Fig. 4: Influence of active constraints on model simplification. Top: Unconstrained, Bottom: Fully constrained.

Besides this, one of the main advantages of considering all the different constraints is the reduction of the error induced by the model simplifications, since an appropriate selection of the input constraints can lead to similar acceleration and deceleration patterns to those obtained with the full nonlinear model. In order to illustrate this, we obtained the optimal speed profile for the second Eco-Trip numerically to incorporate the speed-dependent terms in (2). Here, the limit $v_{\max }$ is imposed with an exterior penalty function $F_{p}(v)=\max \left(0, v(t)-v_{\max }\right)^{2}$ while considering the same final time as in the analytical solution. The comparison between the two speed trajectories is shown in Fig. 4, where the variation during the unconstrained case is $2.51 \%$ while in the fastest trip is only $2.16 \cdot 10^{-4} \%$, which can be neglected. Additionally, some inefficient control actions, like the ones reported in [12], can be avoided since $t_{f}$ complies with the accelerations patterns induced by the input constraints.

\section{ECO-DRIVING CYCLES}

As stated in Section I, the goal of this work was to obtain a method for the computation of driving cycles in a more efficient way and with real-time implementation capabilities compared to the Dynamic Programming developed in [7]. To this end, we first present the considerations adopted for the generation of Eco-Driving cycles and afterwards, a comparison between the solutions given by DP and the proposed approach in an offline setting is provided for different standard cycles.

\section{A. Speed Limits}

In this study, the maximum speeds are defined following a "Standard" legal format [1], resulting in piece-wise constant speed limits as a function of position. Similarly to [7], we consider a sequence $v_{l i m}=\left\{v_{l i m, 1}, v_{l i m, 2}, \ldots, v_{l i m, J}\right\}$ given by the legal restrictions and the maximum speed limits are determined in two steps based on a reference cycle $\left(v_{r e f}\right)$ :

1) Starting from $j=2$, find the index $j \in J$ such that $v_{\text {lim }}(j-1)<v_{\text {ref }}(s(t))$ and $v_{\text {lim }}(j) \geq v_{\text {ref }}(s(t))$

2) Set $v_{\max }(s(t))=v_{\text {lim }}(j)$

Thanks to the current mapping services and traffic monitoring technology, similar speed limits could be obtained as a combination of the legal limits and the current traffic situation for a more practical online implementation.

\section{B. Sub-Trip Segmentation}

Since the analytical expressions consider constant speed limits, we adopted the methodology proposed in [6] to define the boundary conditions, where a set of breaking points is used to divide a longer cycle into smaller sub-trips. Here, the divisions are established in terms of position rather than time given that the characteristics of the driving corridors are generally based on distance [8]. Also, this difference leverages the flexibility of the free final time solutions and makes it possible to define the final speed conditions based on static or long-term road conditions. With this in mind, the boundary conditions for each sub-trip are defined by the speed limits or the stops in a particular cycle such that:

$$
v_{f}(i)= \begin{cases}v_{\max }(i) & \text { if } v_{\max }(i) \leq v_{\max }(i+1) \\ 0 & \text { if } v_{\text {ref }}(i)=0 \\ v_{\max }(i+1) & \text { otherwise }\end{cases}
$$

where $i$ is the position where the breaking point is located. Additionally, short intermediate sub-trips appearing due to consecutive speed limit changes in less than 30 meters are removed to avoid infeasible accelerations or brakings.

\section{Approximations to Dynamic Programming}

1) Penalty Term Definition: In principle, an appropriate definition of the parameter $\beta$ is one of the main contributors to obtain significant energy savings given that with a longer trip time the vehicle can be driven at a lower speed, thus reducing the energy consumption. This improvement is also influenced by the softer acceleration patterns due to the parabolic shape of the speed profiles. For this reason, the heuristic approach in [8] is modified to reflect the wheel-todistance consumption [5], [6] and provide an intuitive way to define the time penalty in (7) for a given driving cycle. This results in the expression

$$
\begin{gathered}
W t D_{C}:=a_{0}+a_{1} \bar{v}+a_{2} \bar{v}^{2}+\frac{\beta}{\bar{v}} \\
\frac{d}{d \bar{v}}\left(W t D_{C}\right)=0 \rightarrow \beta=\bar{v}^{2}\left(a_{1}+2 a_{2} \bar{v}\right)
\end{gathered}
$$

where $\bar{v}=\bar{v}_{\text {ref }}+\epsilon, \bar{v}_{\text {ref }}$ is the mean speed value of the original driving cycle, $\epsilon$ is a separate tuning parameter which modifies the mean speed to meet the desired performance in the ED cycle and the coefficients $a_{n}$ with $n \in\{0,1,2\}$ correspond to the road load equation (2).

2) Optimal Cruise Speed Definition: Another relevant point lost in the analytical solution is the effect of the aerodynamic drag on the optimal speed profile. Specifically, the absence of speed related terms in $(5 \mathrm{~b})$ makes the resulting trajectories reach a higher speed and reduces the energy savings. In contrast, the DP solution tends to maintain a particular cruise speed whenever the speed limits are not reached, which has a large impact on the overall consumption during a driving cycle. For this reason, we included an externally defined Optimal Cruise Speed, denoted by $v_{\text {opt.c }}$, to mitigate the effects of the model simplifications and approximate the trajectories to the DP results. This is done with the help of what we refer to as the motor-to-distance 
TABLE I: Energy Savings [\%]

\begin{tabular}{|c|cccc|}
\hline Driving Cycle & EUDC & NEDC & WLTC & Artemis \\
\hline \hline Analytical & 7.47 & 16.65 & 29.34 & 17.01 \\
Dynamic P. & 8.26 & 21.91 & 31.51 & 18.17 \\
Reduction & 0.86 & 6.73 & 3.16 & 1.41 \\
\hline
\end{tabular}

TABLE II: Difference in SOC [\%]

\begin{tabular}{|c|cccc|}
\hline Driving Cycle & EUDC & NEDC & WLTC & Artemis \\
\hline \hline Original & 2.76 & 4 & 9.24 & 5.82 \\
Analytical & 2.57 & 3.39 & 6.77 & 5.42 \\
Dynamic P. & 2.54 & 3.07 & 6.48 & 5.19 \\
Reduction & 14.53 & 34.13 & 10.32 & 24.35 \\
\hline
\end{tabular}

consumption, which is equivalent to the tank-to-distance consumption in [5], [6]. In this case, the value of $v_{\text {opt.c }}$ is determined with the data of the efficiency map, $P_{E M}(\omega, T)$, instead of using the consumption model (4), such that

$$
v_{\text {opt.c }}=\underset{v}{\arg \min }\left(\frac{P_{E M}(\tilde{\omega}, \tilde{T})+\beta z}{\tilde{v}}\right)
$$

Here, $\{\tilde{T}, \tilde{\omega}, \tilde{v}\}$ are torque, angular speed and longitudinal speed vectors corresponding to constant speed driving that take the complete vehicle dynamic model into consideration while using a fine grid of values between $[1,130][\mathrm{km} / \mathrm{h}]$. Also, $z$ is a scaling factor relating $\beta$ and $v_{\text {opt.c. }}$.

Once the optimal cruise speed is defined for a particular cycle, all the speed limits higher than $v_{\text {opt.c }}$ are replaced by the optimal cruise speed to serve as constant speed periods tailored for the trip at hand and avoid the large energy usage incurred for driving at high speeds.

\section{Simulation Results}

In this section, the energy savings obtained with the proposed approach in an offline way are evaluated and compared to the global optimal solution given by DP. The resulting speed profiles, the energy savings, final SOC and the operating points in the motor efficiency map are considered to analyze the differences between both methods. To this end, four different standard driving cycles are selected as the reference speeds to evaluate the potential energy savings, namely, the EUDC (Extra-Urban Driving Cycle), NEDC (New European Driving Cycle), WLTC (Worldwide harmonized Light vehicles Test Cycle) and the Artemis Road cycle [15]. Also, we defined $v_{\text {lim }}=\{0,35,50,70,100,130\}$ as the speed limit vector for all the tests based on the NEDC. Even though this vector can be freely chosen, it is worth mentioning that its relation with the final speed condition has a significant influence on the resulting profile, since unnecessary sub-trip trajectories could be imposed. To provide a fair comparison, the full vehicle model and the electric motor map are used to obtain the torque, SOC and energy trajectories required to follow each speed profile and remove the influence of the simplifications. Also, the parameter $\epsilon$ related to the mean speed in (21) is tuned such that the difference in trip duration between the analytical and DP cycles is less than $0.2 \%$, removing the trade-off between energetic performance and trip time. Besides, the state and

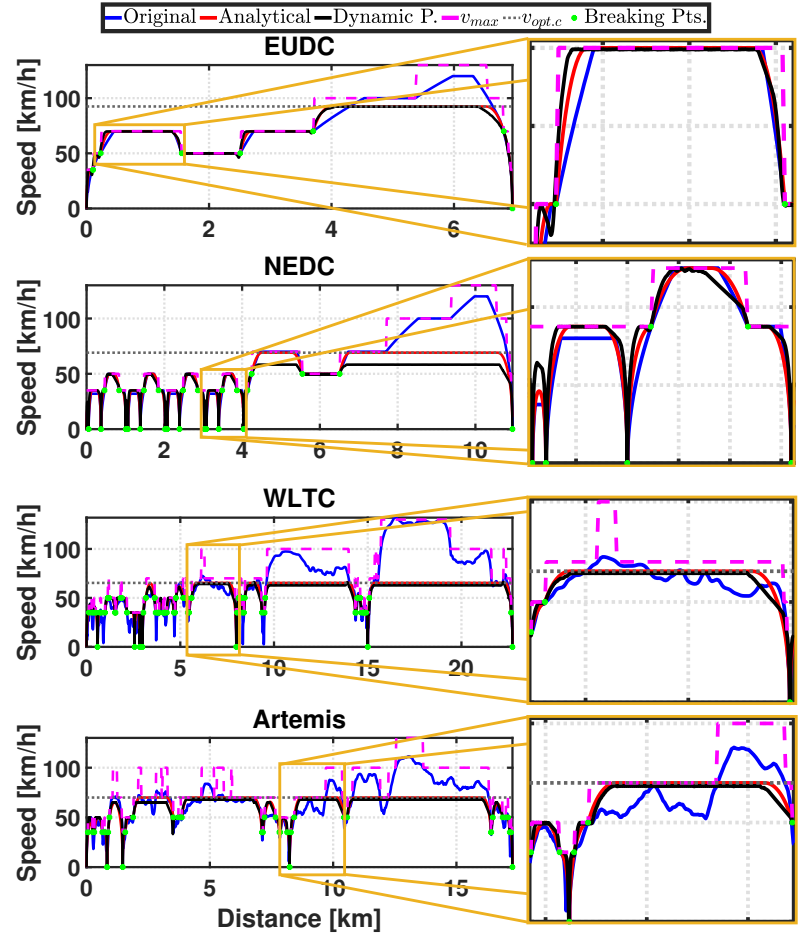

Fig. 5: Comparison of speed profiles.

input discretization steps for DP are specified as $\delta s=4[\mathrm{~m}]$, $\delta v=0.02[\mathrm{~m} / \mathrm{s}]$ and $\delta u=0.5[\mathrm{Nm}]$.

Even though the analytical and DP trajectories shown in Fig. 5 are quite similar, some recurrent patterns causing most of the differences in energy savings can be observed. First of all, the fact that DP uses information about the entire cycle to optimally balance the energy consumption results in fast accelerations during low velocity sections to save time and reduce the aerodynamic drag losses for driving at high speeds. In contrast, the driving behaviour in the analytical approach only depends on the time penalty specified and the information embedded in the boundary conditions, the input limits and the top speed for each sub-trip regardless of the point at which the vehicle is located with respect to the cycle.

A second and more relevant characteristic is the presence of a coasting mode, where shorter constant speed segments are obtained with DP due to the distance covered without applying any input, which leads to a reduced consumption as presented in Table I. This feature is lost in the analytical solution due to the parabolic form of the trajectories and the modeling simplifications, affecting the modulation of

TABLE III: Final Time [s]

\begin{tabular}{|c|cccc|}
\hline Driving Cycle & EUDC & NEDC & WLTC & Artemis \\
\hline \hline Original & 360 & 1149 & 1785 & 1073 \\
Analytical & 360.95 & 1151.52 & 1783.63 & 1077.72 \\
Dynamic P. & 360.95 & 1151.53 & 1783.34 & 1076.12 \\
\hline
\end{tabular}

TABLE IV: Time [s] to compute the eco-cycles

\begin{tabular}{|c|cccc|}
\hline Driving Cycle & EUDC & NEDC & WLTC & Artemis \\
\hline \hline Analytical & 0.046 & 0.080 & 0.096 & 0.091 \\
Dynamic P. & 119.60 & 188.17 & 382.04 & 118.44 \\
Improvement & $\times 2600$ & $\times 2352.1$ & $\times 3979.6$ & $\times 1301.5$ \\
\hline
\end{tabular}



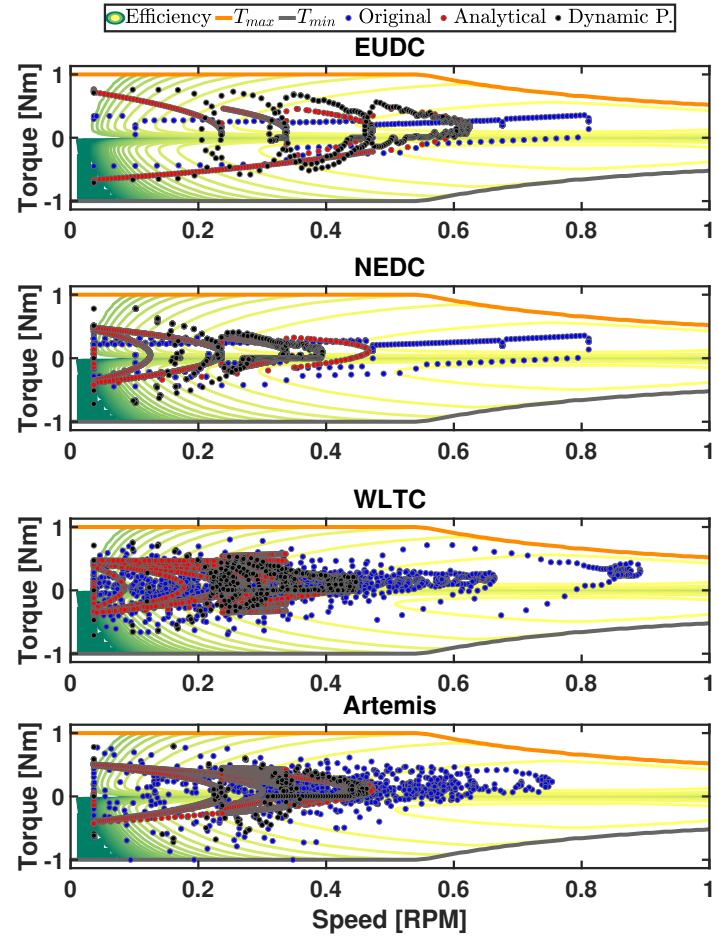

Fig. 6: Comparison of normalized operating points.

the braking patterns and thus, the energy recovered through regeneration. Table II contains the difference in SOC for each cycle and the relative reduction of the analytical solution with respect to DP, where the influence of the constant speed periods imposed by $v_{\text {opt.c }}$ becomes more evident since a bigger cruise speed variation causes a larger SOC reduction.

In general, these differences are more evident in cycles with multiple stops like the NEDC, where DP tends to go as fast as possible during the low speed regions to maintain a lower cruise speed after the $4^{\text {th }}$ kilometer of the cycle. Conversely, the analytical profile needs to go about $10[\mathrm{~km} / \mathrm{h}]$ faster to reach the final position at the same time, which increases the energy losses as depicted by the operating points in Fig. 6. On the other hand, if the original cycle travels longer distances with fewer stops (see the WLTC and Artemis trajectories in Fig. 5) or even without them, e.g., EUDC in Fig. 5, the speed profiles become almost identical and the only meaningful differences are the zero input periods shown in Fig. 6, i.e., coasting mode. However, the main benefit of the analytical approach over DP is its potential for a real-time implementation thanks to the significant reduction in computation time (see Table IV).

\section{CONCLUSION}

In this paper, a continuous-time optimal control problem to obtain an Eco-Driving strategy for electric vehicles with free final time was formulated. We presented a systematic approach based on Pontryagin's Minimum Principle to solve the problem analytically and different closed-form solutions were developed depending on the constraints that become active during a trip. A simple strategy was developed for the computation of ED cycles with a reduced complexity and real-time capabilities, where the proposed approach is more than 2550 times faster than Dynamic Programming, on average. Nonetheless, this improvement comes at the cost of losing global optimality of the solution due to the simplifications required. For this reason, a method to specify the optimal cruise speed was proposed, which helped to achieve between $93.27 \%$ and $99.14 \%$ of the optimal energy savings when input and speed limits were incorporated.

Current research focuses on the extension of these closedform solutions for conventional and hybrid vehicles while considering the incorporation of coasting phases to achieve softer and more efficient decelerations. Furthermore, alternative strategies to define the final speed conditions as well as feasibility restrictions for each driving mode sequence are being explored to improve the performance and robustness of the solutions while leveraging their low computational complexity in a receding or shrinking horizon formulation.

\section{REFERENCES}

[1] F. Mensing, E. Bideaux, R. Trigui, J. Ribet, and B. Jeanneret, "Ecodriving: An economic or ecologic driving style?" Transportation Research Part C: Emerging Technologies, vol. 38, p. 110-121, 01 2014.

[2] IEA, "Transport Energy Efficiency," International Energy Agency, Tech. Rep., 2010. [Online]. Available: https://www.iea.org/reports/transport-energy-efficiency

[3] C. Bingham, C. Walsh, and S. Carroll, "Impact of driving characteristics on electric vehicle energy consumption and range," Intelligent Transport Systems, IET, vol. 6, pp. 29-35, 032012.

[4] IEA, "Global EV Outlook 2020," International Energy Agency, Tech. Rep., 2020. [Online]. Available: https://www.iea.org/reports/globalev-outlook-2020

[5] B. Saerens, "Optimal control based eco-driving," Ph.D. dissertation, K. U. Leuven, 062012.

[6] A. Sciarretta and A. Vahidi, Energy-Efficient Driving of Road Vehicles, ser. Lecture Notes in Intelligent Transportation and Infrastructure. Springer International Publishing, 2020.

[7] D. Maamria, K. Gillet, G. Colin, Y. Chamaillard, and C. Nouillant, "On the use of dynamic programming in eco-driving cycle computation for electric vehicles," in 2016 IEEE Conference on Control Applications (CCA), 2016, pp. 1288-1293.

[8] _ , "Optimal predictive eco-driving cycles for conventional, electric, and hybrid electric cars," IEEE Transactions on Vehicular Technology, vol. 68, no. 7, pp. 6320-6330, 2019.

[9] G. Padilla Cazar, S. Weiland, and M. Donkers, "A global optimal solution to the eco-driving problem," IEEE Control Systems Letters, vol. 2, no. 4, pp. 599 - 604, 102018.

[10] J. Flores Paredes, G. Padilla Cazar, and M. Donkers, "A shrinking horizon approach to eco-driving for electric city buses: implementation and experimental results," in IFAC International Symposium on Advances on Automotive Control, ser. IFAC Papers Online, no. 5. Netherlands: Elsevier, 2019, pp. 556-561.

[11] D. Karbowski, J. Jeong, K. Elands, and I. Dobrovolschi, "Modelpredictive eco-driving for electrified connected and automated vehicles," in 32nd Electric Vehicle Symposium (EVS32), 052019

[12] J. Han, A. Sciarretta, L. L. Ojeda, G. De Nunzio, and L. Thibault, "Safe- and eco-driving control for connected and automated electric vehicles using analytical state-constrained optimal solution," IEEE Transactions on Intelligent Vehicles, vol. 3, no. 2, pp. 163-172, 2018.

[13] J. Han, D. Karbowski, and D. Karbowski, "Closed-form solutions for a real-time energy-optimal and collision-free speed planner with limited information," in 2020 American Control Conference (ACC), 2020, pp. 268-275.

[14] B. Chachuat, Nonlinear and Dynamic Optimization: From Theory to Practice, 2007, url: http://lawww.epfl.ch/page4234.html. [Online]. Available: http://infoscience.epfl.ch/record/111939

[15] M. Andre, "The artemis european driving cycles for measuring car pollutant emissions," The Science of the total environment, vol. 334335, pp. 73-84, 012005. 